Article

\title{
Antique Traditional Practice: Phenolic Profile of Virgin Olive Oil Obtained from Fruits Stored in Seawater
}

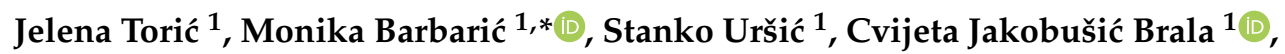 \\ Ana Karković Markovićc ${ }^{1}$, Maja Zebić Avdičević ${ }^{2}$ and Đani Benčić ${ }^{3}$ \\ 1 Faculty of Pharmacy and Biochemistry, University of Zagreb, A. Kovačića 1, 10000 Zagreb, Croatia; \\ jelenatoric@gmail.com (J.T.); dr.sursic@gmail.com (S.U.); cjakobus@pharma.hr (C.J.B.); \\ akarkovic@pharma.hr (A.K.M.) \\ 2 Faculty of Mechanical Engineering and Naval Architecture, University of Zagreb, Ivana Lučića 5, \\ 10000 Zagreb, Croatia; maja.zebic@fsb.hr \\ 3 Faculty of Agriculture, University of Zagreb, Svetošimunska cesta 25, 10000 Zagreb, Croatia; bencic@agr.hr \\ * Correspondence: mbarbaric@pharma.hr; Tel.: +385-01-6394-472; Fax: +385-01-6394-400
}

Received: 25 August 2020; Accepted: 21 September 2020; Published: 23 September 2020

\begin{abstract}
Virgin olive oil (VOO) is a functional food specific to the Mediterranean diet and related to human health, especially as a protector of cardiovascular health, in the prevention of several types of cancers, and in modification of immune and inflammatory response. Phenolic compounds have central importance for these extraordinary health benefits. In the production of high-quality olive oils, it is very important to process freshly picked olives and avoid any storage of fruits. However, in Croatia there is a very traditional and environmentally friendly method of olive oil production, where olive fruits are stored in seawater for some time prior to processing. This practice is also notable nowadays since there are people who prefer the characteristic flavor of the "seawater olive oil", although some people argue against its quality and biomedical relevance. In this study, the phenolic contents of VOO prepared from the immediately processed fresh olives and olives processed after storage in seawater were compared with the use of high-performance liquid chromatography-mass spectrometry (HPLC-MS) and spectrophotometric analysis. The results suggest that "seawater olive oil" should be considered as a safe food of biomedical relevance, as it still contains a significant proportion of important phenolics like hydroxytyrosol, tyrosol and oleacein (e.g., 63.2\% of total phenols in comparison to VOO).
\end{abstract}

Keywords: HPLC analysis; olive oil; phenolic compounds; seawater

\section{Introduction}

Most of the extra virgin olive oils (EVOOs) of the highest quality from the Croatian coastal region and isles of the Adriatic Sea are obtained by pressing freshly picked olives and avoiding any storage of fruits before common mechanical processing. However, although it is well known that immediate processing of olive fruits is an important factor to obtain high quality olive oil, in many cases limited capacities of olive oil mills do not make possible the quick processing of collected olives. A very traditional method of olive oil production in Croatia (the storage of olive fruits in seawater for some time prior to processing into oil) is frequently used in the coastal area and isles. The tradition has persisted in the Adriatic since the Roman Empire [1]. There are people who prefer the characteristic flavor of the "seawater olive oil" (that is, the oil obtained from olives stored for some time in seawater) in contrast to others who have claimed that this olive oil is inferior in any way in comparison with oils obtained using a common modern practice. 
Notwithstanding, due to differences in flavor it is customary to have both types of the olive oils at the table frequently, and additionally owing to recently awakened interests of visitors and tourists in the traditionally obtained olive oil, there is an increased demand in the local market for the oil from the seawater stored olives.

Virgin olive oils (VOOs), as is very well known, are not just nutritious but also have health benefits. Many studies suggesting numerous beneficial health effects associated with dietary consumption of EVOOs have steadily accumulated for decades. In this context, to mention only a few issues, the phenolic compounds naturally present in EVOOs are deemed to be of central importance for the beneficial cardiovascular, antiatherogenic/anti-inflammatory, anticancer, neuroprotective, antioxidant and antimicrobial effects [2-8].

Therefore, when dealing with the differences between the oils from seawater stored olives and the oils obtained using a common modern practice, there is an important question as to whether the phenolic compounds naturally present in olive fruits (and consequently in olive oil obtained by common practice) have been preserved or lost in the "seawater oils", at least to some extent during the storage in seawater. Intuitively, one would expect that the phenolics contained in olive fruits could be lost extensively due to enzymatic processes expected to take place during the seawater storage. However, to the best of our knowledge there is no study dealing with the question to date. Therefore, to obtain quantitative data needed to reach an informed and reliable insight into the issue, we performed a side-by-side analysis of the phenolic content in the olive oil prepared from fresh olive fruits immediately after harvest (VOO) and the "seawater olive oil" (VOO-Sea). The oils were obtained using samples of olive fruits from a batch of the same olive trees of the variety Oblica harvested on the Ugljan isle (central part of Croatian Adriatic, nearby the city of Zadar) in the 2016 season. Oblica is the main variety in the Dalmatian region spread throughout the Mediterranean area of Croatia where it occupies about $60 \%$ of all productional olive trees [9]. It should be noted that the Isle of Ugljan was an important point in the olive oil production during the Roman Empire age, continuing up to the olive cultivation and oil production to date.

\section{Materials and Methods}

\subsection{Reagents and Standards}

Methanol (high-performance liquid chromatography, HPLC grade), 2,2-diphenyl-1-picrylhydrazyl (DPPH), catechin, benzoic acid, hydroxytyrosol, oleuropein, homovanillyl alcohol, $p$-coumaric acid, pinoresinol and apigenin were purchased from Sigma-Aldrich Chemie $\mathrm{GmbH}$ (Steinheim, Germany). Tyrosol, 3,4-dihydroxybenzoic acid, gallic acid, $p$-hydroxybenzoic acid, syringic acid, vanillic acid, cinnamic acid, ferulic acid, $o$-coumaric acid and Folin-Ciocalteu's reagent were obtained from Fluka Chemie GmbH (Buchs, Switzerland). Formic acid (99+\%) and vanillin were purchased from Acros Organics (Morris Plains, NJ, USA). Sodium hydroxide and sodium carbonate anhydrous were purchased from Kemika (Zagreb, Croatia) while sodium molibdate dehydrate, sodium nitrite, glacial acetic acid and aluminum chloride were obtained from Merck (Darmstadt, Germany).

\subsection{Olive Oil Samples Preparation}

The olive oil samples were obtained using olive fruits from a batch of the same olive trees of the variety Oblica. All olive trees were grown under the same agronomic and environmental conditions on the Ugljan isle (central part of Croatian Adriatic, nearby the city of Zadar). After harvesting in the 2016 season, fresh olive fruits $(50.0 \mathrm{~kg}$ ) were immediately processed by centrifugal line (Olimio, $150 \mathrm{~kg}$, Enologia Mori, Tuscany, Italy) giving virgin olive oil (VOO). The other fresh olive fruits (50.0 kg) were kept in the seawater for ten days and then removed from the seawater and processed on the same line giving "seawater olive oil" (VOO-Sea). All samples were transferred in dark glass bottles without headspace and stored in the dark at $4{ }^{\circ} \mathrm{C}$ until analysis. 


\subsection{Phenolic Compounds}

\subsubsection{Extraction}

Extractions of phenolic compounds from VOO and VOO-Sea were carried out using the ultrasound-assisted liquid-liquid extraction (US-LLE) method previously described by Jerman Klen and Mozetič Vodopivec [10] with some modifications. VOO and VOO-Sea (20 g) were dissolved in $n$-hexane $(10 \mathrm{~mL})$ and sonicated $\left(3 \times 10 \mathrm{~min}\right.$ at $\left.25^{\circ} \mathrm{C}\right)$ with methanol $(15 \mathrm{~mL})$ using an ultrasound bath (Elma Transsonic T570 HF $=320 \mathrm{~W}$, Singen, Germany). The homogenates of each extraction step were centrifuged (15 min at $4000 \mathrm{rpm}$ ) using Hettich zentrifugen D-78532 (Tuttlingen, Germany), combined and defatted by shaking the suspension with hexane. The methanolic extracts were concentrated with rotary evaporator (Büchi Heating Bath B-490, Büchi Labortechnik AG, Flawil, Switzerland) at $38{ }^{\circ} \mathrm{C}$. After successive methanol evaporation, the residue was redissolved in methanol $(1.7 \mathrm{~mL})$ and filtered through $0.20 \mu \mathrm{m} / 13 \mathrm{~mm}$ polytetrafluoroethylen (PTFE) filters (Macherey-Nagel GmbH \& Co. KG, Düren, Germany) prior to analyses. Each sample of oil was extracted three times.

\subsubsection{HPLC-DAD Analysis}

A HPLC-DAD analysis was performed essentially by applying the procedure [11]. The HPLC system (Perkin Elmer Series 200, Shelton, CT, USA) equipped with diode array detector (DAD) was used. Separation was achieved at $25^{\circ} \mathrm{C}$ on a C18 Restek (Bellefonte, PA, USA) column $(5 \mu \mathrm{m}, 250 \times 4 \mathrm{~mm})$. The flow rate was $1 \mathrm{~mL} / \mathrm{min}$, using $25 \mu \mathrm{L}$ of injection volume and a solvent system composed of water/acetic acid (98:2, v/v) (A) and methanol (B) for a total running time of $45 \mathrm{~min}$. The gradient elution was as follows: $0-2$ min at 5\% B (initial conditions); $2-8$ min linear gradient from 5\% B to $25 \% \mathrm{~B}$; 10-20 min at 40\% B; 20-40 min linear gradient from $40 \% \mathrm{~B}$ to $50 \% \mathrm{~B}$; and $0 \% \mathrm{~A}-100 \% \mathrm{~B}$ until the end of the run.

The UV absorption of eluates at $278 \mathrm{~nm}$ was recorded. The phenolic compounds were identified by comparing their retention times $\left(R_{\mathrm{t}}\right)$ with those of the standards (Table S1 in Supplementary Materials). Oleacein (dialdehydic form of decarboxymethyl elenolic acid linked to hydroxytyrosol, 3,4-DHPEA-EDA) was identified tentatively according to the literature and confirmed by mass spectrometry (MS) analysis. Quantification of identified phenolic compounds was carried out by the integrated peak areas using the appropriate calibration curves with authentic standards (Table S1 in Supplementary Materials) in all cases except oleacein. Actual concentration of oleacein was calculated from the calibration curve for oleuropein, taking into account the differences in their molecular weights [12]. Phenolic compound content was expressed as $\mathrm{mg}$ of phenol/kg of VOO or VOO-Sea.

\subsubsection{MS Analysis}

HPLC-MS analysis was carried out using a HPLC Agilent Technologies 1200 Series (Santa Clara, CA, USA) with binary pump, degasser, autosampler and DAD attached to Agilent Technologies 6420 Triple Quad LC/MS spectrometer equipped with electrospray ionization (ESI) source. Chromatographic separation was achieved using a C18 reversed-phase analytical column, Kinetex C18 $(2.1 \times 50 \mathrm{~mm}$, $1.7 \mu \mathrm{m}$ particle size, $100 \AA$ A, Phenomenex, Torrance, CA, USA). The flow rate was set at $0.5 \mathrm{~mL} / \mathrm{min}$, and the sample injection volume was $10 \mu \mathrm{L}$. The elution gradient consisted of mobile phase water/formic acid (99.9:0.1, v/v) (A) and methanol (B) for a total running time of $80 \mathrm{~min}$. The gradient elution was as follows: $0-0.5 \mathrm{~min}$ at $90 \% \mathrm{~A} ; 0.5-74.5 \mathrm{~min}$ linear gradient from $10 \% \mathrm{~B}$ to $90 \% \mathrm{~B}$; and $90 \% \mathrm{~A}-10 \% \mathrm{~B}$ until the end of the run.

MS analysis was performed using a heated ESI source operating in negative ion modes (NIM) in the range $m / z$ 50-2000 Da. The ESI needle voltage was held at ground and the capillary voltage (Vcap) set at $3500 \mathrm{~V}$. Source conditions were as follows: nebuliser, 8 psi; drying gas temperature, $300{ }^{\circ} \mathrm{C}$; drying gas flow, $8 \mathrm{~L} / \mathrm{min}$ and fragmentor, $135 \mathrm{~V}$. Nitrogen was used as source gas and as collision gas. The identity of oleacein, oleocanthal, oleuropein aglycone and ligstroside aglycone were confirmed by ESI-MS fragmentation profile of molecular $[\mathrm{M}-\mathrm{H}]^{-}$ions in comparison with those from the literature. 


\subsubsection{Spectrophotometric Analysis}

Total Phenols (TP)

TP (total phenols) concentrations in the VOO and VOO-Sea were determined spectrophotometrically at $725 \mathrm{~nm}$ using Folin-Ciocalteu (FC) reagent, according to Gutfinger [13]. Each sample of phenolic extract was analyzed two times. Aliquots of phenolic extract (PE) and PE-Sea were transferred to $10 \mathrm{~mL}$ volumetric flasks, and then water $(5 \mathrm{~mL})$ and $\mathrm{FC}$ reagent $(0.25 \mathrm{~mL})$ was added. The solution of saturated $(20 \%)$ sodium carbonate $(1.5 \mathrm{~mL})$ was added to the reaction mixture after $3 \mathrm{~min}$. The solution was then diluted with water to $10 \mathrm{~mL}$. After $30 \mathrm{~min}$, the absorbance was measured on UV-VIS spectrophotometer (Hewlett Packard 8453, Böblingen, Germany) at $725 \mathrm{~nm}$ against a methanol blank. Gallic acid was used as a standard for preparing the calibration curve (Table S2 in Supplementary Materials) and the amount of TP in extracts was expressed as mg gallic acid equivalent (GAE)/kg of VOO or VOO-Sea.

o-Diphenols

The concentration of $o$-diphenols in VOO and VOO-Sea was determined by sodium molybdate assay [14]. Each sample of phenolic extract was analyzed two times. An aliquote of $0.5 \mathrm{~mL}$ of extract was diluted with $5 \mathrm{~mL}$ methanol/water $(1: 1, v / v)$ to prepare a diluted extract. Then, $0.5 \mathrm{~mL}$ of a $5 \%$ sodium molibdate solution in methanol/water $(1: 1, v / v)$ was added in $2 \mathrm{~mL}$ of diluted extract. The content was mixed and kept in the dark $(15 \mathrm{~min})$ and then the absorbance was measured at $350 \mathrm{~nm}$ spectrophotometrically against a reagent blank. A calibration curve (Table S2 in Supplementary Materials) was obtained by measurement of standard solutions of gallic acid following the procedure described above. The concentration of $o$-diphenols in extracts was expressed as $\mathrm{mg}$ gallic acid equivalent (GAE)/kg of VOO or VOO-Sea.

Total Flavonoids (TF)

TF concentration in VOO and VOO-Sea was determined by spectrophotometric assay [15]. Each sample of phenolic extract was analyzed two times. A $1 \mathrm{~mL}$ aliquot of appropriately diluted extracts was added to a $10 \mathrm{~mL}$ volumetric flask containing $4 \mathrm{~mL}$ water. Then, a $5 \%$ sodium nitrite solution $(0.3 \mathrm{~mL})$ was added, followed by a $10 \%$ aluminum chloride solution $(0.3 \mathrm{~mL})$. Test tubes were incubated at ambient temperature for $5 \mathrm{~min}$, and then $2 \mathrm{~mL}$ of $1 \mathrm{M}$ sodium hydroxide was added to the mixture. The volume was immediately adjusted to $10 \mathrm{~mL}$ with water and thoroughly mixed. Absorbance of the mixture, pink in color, was determined at $510 \mathrm{~nm}$. Catechin served as a standard for preparation of the calibration curve (Table S2 in Supplementary Materials). The concentration of TF was expressed as $\mathrm{mg}$ catechin equivalents $(\mathrm{CE}) / \mathrm{kg}$ of VOO or VOO-Sea.

\section{DPPH Test of Antioxidant Activity}

The phenolic extracts, PE and PE-Sea, were analyzed according to the procedure described by Villaño et al. [16] with some modifications. Aliquots $(0.1 \mathrm{~mL})$ of five different concentrations of diluted PEs were added to methanolic solution of DPPH radical $\left(2.9 \mathrm{~mL}, 7.5 \times 10^{-5} \mathrm{M}\right)$. The solution was shaken, and after 30 min kept in dark, the absorbance at $517 \mathrm{~nm}$ was measured. DPPH scavenging effect was calculated according to the equation: $\% \mathrm{DPPH}$ scavenging effect $=\left(A_{\mathrm{DPPH}}-A_{\text {sample }}\right) / A_{\mathrm{DPPH}}$ $\times 100$, with $A_{\mathrm{DPPH}}$ is the absorbance of DPPH solution, $A_{\text {sample }}$ is the absorbance of the examined solution $(\mathrm{DPPH}+\mathrm{PE})$. The $\mathrm{EC}_{50}$ value is the concentration of TP in PE or PE-Sea ( $\mu \mathrm{g}$ GAE $/ \mathrm{mL}$ PE or PE-Sea, corresponding to $50 \%$ reduction of the initial DPPH concentration), and was obtained from the dependence of the \% scavenging effect on the concentration of PE. 


\subsection{Statistical Analysis}

Analysis of variance (ANOVA) test $(n=3)$, Pearson's correlation tests $(p<0.01 ; p<0.05)$ and principal component analysis (PCA) were performed in Python 2.7. software package using SciPy.stats library (Python Software Foundation, Beaverton, OR, USA).

\section{Results and Discussion}

\subsection{Identification and Quantification of Phenolic Compounds}

In this study the comparison of the phenolic profile of VOO prepared from immediately processed fresh olive fruits, and VOO-Sea prepared from fruits that were kept in seawater for ten days before processing, was performed. Olive fruits were collected from the same olive trees of the variety Oblica from the Ugljan isle (central part of Croatian Adriatic, near to the city of Zadar) in the 2016 season.

The phenolic profiles of the oils after extraction of phenolic compounds by optimized US-LLE method (Section 2.3.1) were analyzed using different methods (Section 2.3.2, Section 2.3.3, Section 2.3.4). The HPLC-DAD analysis of phenolic extracts showed several peaks corresponding to different phenolics (Figures 1 and 2) determined under the chromatographic conditions described above (Section 2.3.2). Those peaks correspond to the important bioactive compounds hydroxytyrosol, tyrosol and oleacein and another 11 minor phenolic compounds. Phenolic acids like 3,4-dihydroxybenzoic acid, o-coumaric acid and syringic acid were below the limit of detection in VOO and VOO-Sea.

Oleacein (peak 10), the most abundant secoiridoid in all analyzed oil samples, was firstly identified by its $R_{\mathrm{t}}$ tentatively according to the literature [17]. Identity of oleacein was confirmed by MS analysis (Section 2.3.3). Analysis of fragmentation of molecules in ESI-MS spectra (Figures S1 and S2 in Supplementary Materials) also confirmed the presence of other bioactive secoiridoids (oleocanthal, oleuropein aglycone and ligstroside aglycone) in VOO and VOO-Sea but quantification was not possible (peaks were not resolved). The extracted-ion chromatograms (EICs) generated for PE (Figure S1b in Supplementary material) and PE-Sea (Figure S2b in Supplementary Materials), gave the deprotonated molecule at $m / z 639,319,195,183,165,59$ which demonstrates the presence of oleacein [18]. The EIC chromatograms on Figures S1c and S2c showed $m / z$ 607, 361, 303, 291, 285, 259, 179, 165, 139 in full-scan mode. According to the literature, these $m / z$ 361, 291, 259, 139 peaks may correspond to ligstroside aglycone isomers (ligstroside aglycone mono-aldehyde; $p$-HPEA-EA) and $m / z$ 607, 303, $285,179,165$ peaks correspond to open ring decarboxylated dialdehidic form of ligstroside derivative, oleocanthal (dialdehydic form of decarboxymethyl elenolic acid linked to tyrosol; $p$-HPEA-EDA) [18]. Figures S1d and S2d show EIC chromatograms of PE and PE-Sea with fragmentation $m / z$ 755, 377, $345,307,275,139$ which correspond to oleuropein aglycone (oleuropein aglycone mono-aldehyde, 3,4-DHPEA-EA) [18].

The phenolic compound concentrations ( $\mathrm{mg} / \mathrm{kg}$ VOO or VOO-Sea) are shown in Table 1.

The determined concentrations of hydroxytyrosol in VOO and VOO-Sea, that amount to $18.2 \mathrm{mg} / \mathrm{kg}$ and $12.9 \mathrm{mg} / \mathrm{kg}$, respectively, are generally in the range similar to the values in some Spanish $(6.9-72.7 \mathrm{mg} / \mathrm{kg}$ of oil) or Italian EVOOs $(0.85-36.7 \mathrm{mg} / \mathrm{kg}$ of oil) $[17,19,20]$, and are even higher than those previously published for Oblica variety $(6.2-11.3 \mathrm{mg} / \mathrm{kg}$ of oil) $[11,21,22]$. Furthermore, the tyrosol concentration determined in VOO and VOO-Sea $(8.10 \mathrm{mg} / \mathrm{kg}$ and $7.20 \mathrm{mg} / \mathrm{kg}$ respectively) is very similar to the values in the same literature.

The concentrations of total phenols, $o$-diphenols, total flavonoids and $\mathrm{EC}_{50}$ values of phenolic extracts were determined spectrophotometrically and are summarized in Table 2 . The values of TP and $o$-diphenols found in VOO and VOO-Sea are frequently greater in comparison with the values of $\mathrm{TP}$ and $o$-diphenols in VOOs from other countries [23-27]. 


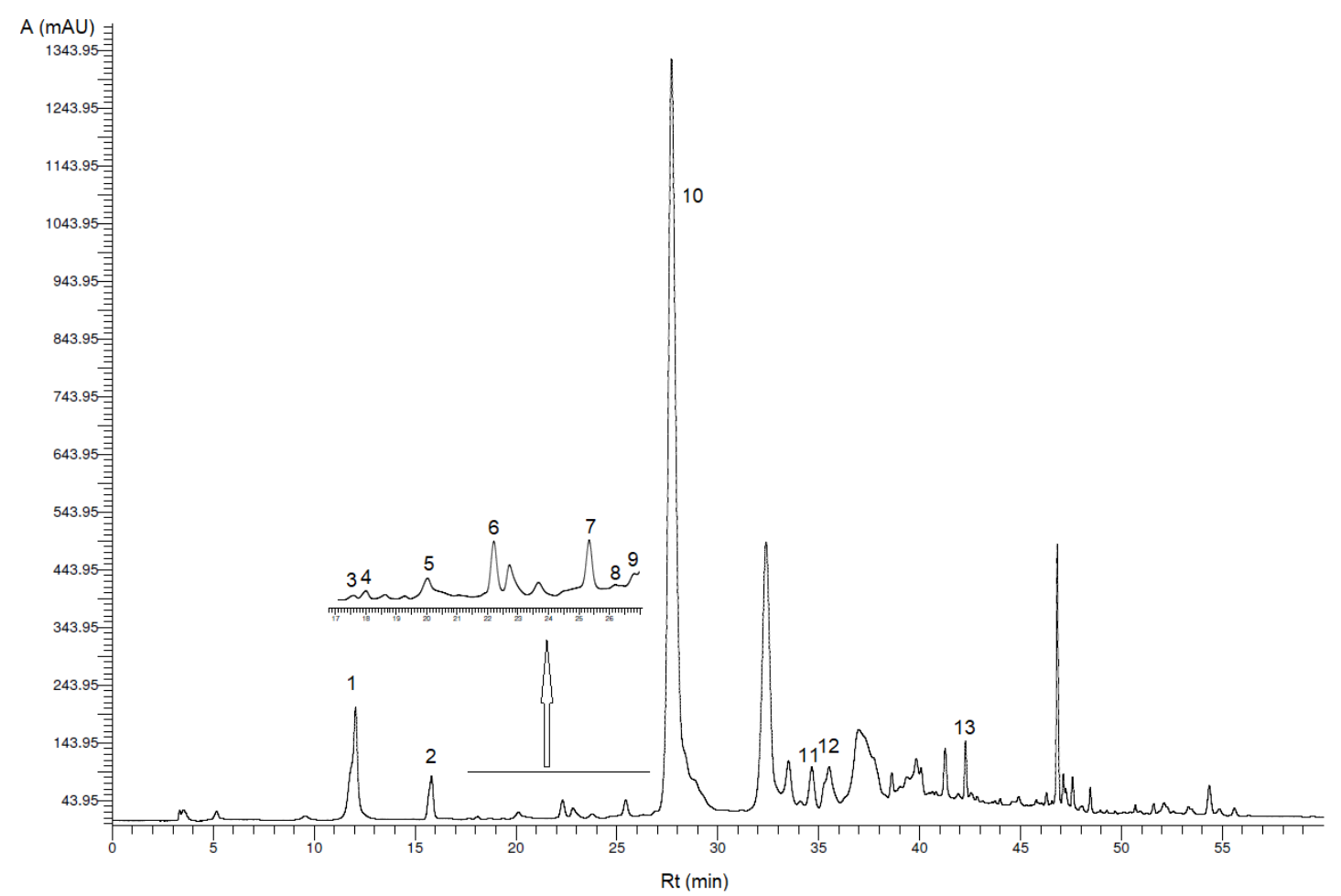

Figure 1. High-performance liquid chromatography (HPLC) chromatogram of PE (phenolic extract) at $278 \mathrm{~nm}$. Peaks correspond to phenolic compounds in Table 1 (A-absorbance, $R_{t}$-retention time).

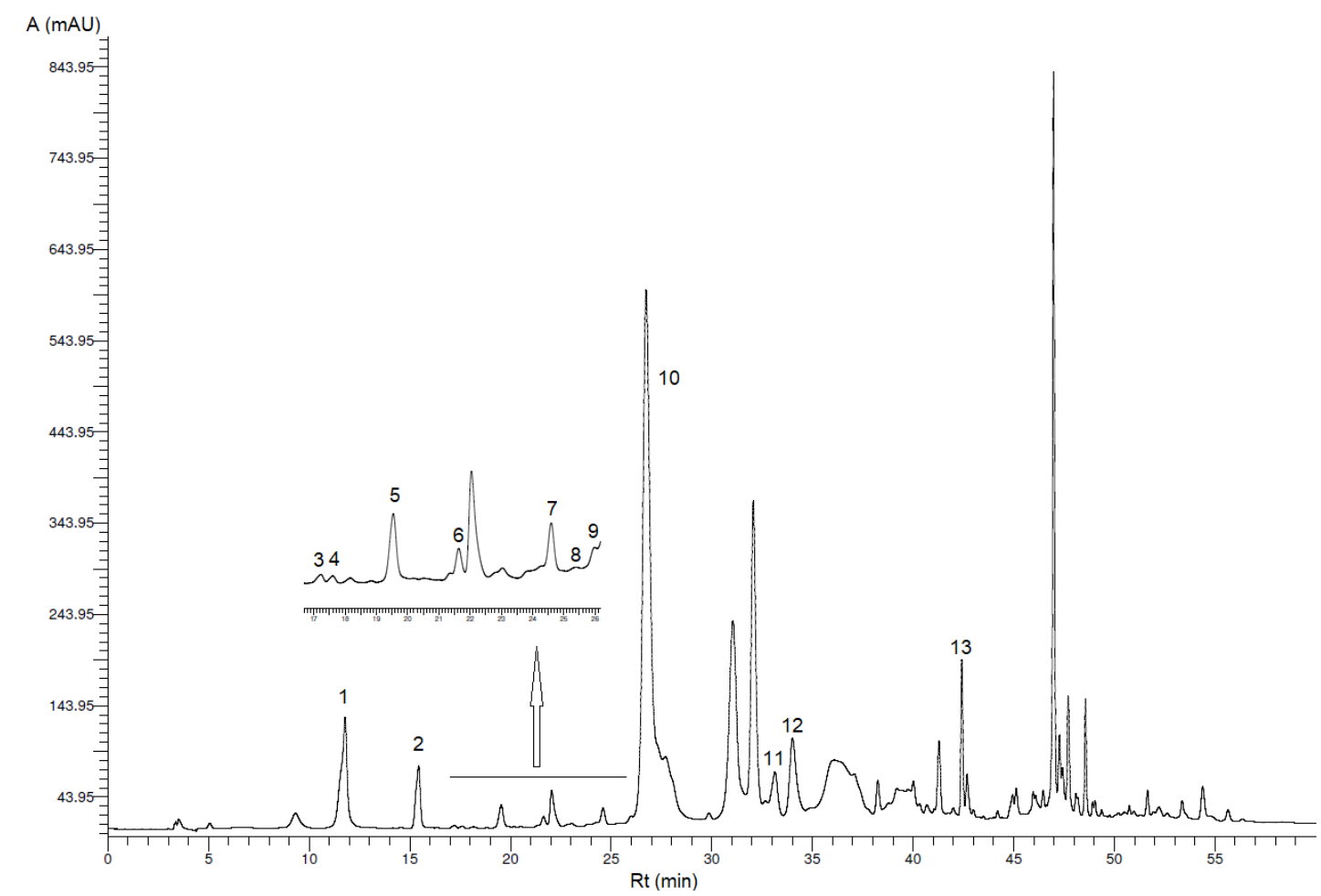

Figure 2. HPLC chromatogram of PE-Sea at $278 \mathrm{~nm}$. Peaks correspond to phenolic compounds in Table 1 (A-absorbance, $R_{t}$-retention time). 
Table 1. Concentrations of phenolic compounds (mean value $\pm \mathrm{SD} ; n=3$ ) in VOO (virgin olive oil) and VOO-Sea (virgin seawater-stored olive oil) determined by high-performance liquid chromatography-diode array detector (HPLC-DAD) analysis.

\begin{tabular}{|c|c|c|c|}
\hline Peak * & Phenolic Compound & VOO $(\mathrm{mg} / \mathrm{kg}$ VOO $\pm \mathrm{SD})$ & VOO-Sea $(\mathrm{mg} / \mathrm{kg}$ VOO-Sea $\pm \mathrm{SD})$ \\
\hline \multirow[t]{2}{*}{1} & Hydroxytyrosol & $18.2^{b} \pm 3.0$ & $12.9^{\mathrm{a}} \pm 1.0$ \\
\hline & 3,4-Dihydroxybenzoic acid & n.d. & n.d. \\
\hline 2 & Tyrosol & $8.10^{b} \pm 0.34$ & $7.20^{a} \pm 0.28$ \\
\hline 3 & p-Hydroxybenzoic acid & $0.10 \pm 0.02 * *$ & $0.11 \pm 0.00^{* *}$ \\
\hline 4 & Homovanillyl alcohol & $0.38 \pm 0.04^{* *}$ & $0.22 \pm 0.01^{* *}$ \\
\hline \multirow[t]{2}{*}{5} & Vanillic acid & $0.64^{a} \pm 0.20$ & $0.97^{b} \pm 0.03$ \\
\hline & Syringic acid & n.d. & n.d. \\
\hline 6 & Vanillin & $0.53 \pm 0.05$ & $0.23 \pm 0.01^{* *}$ \\
\hline 7 & $p$-Coumaric acid & $0.43 \pm 0.04^{* *}$ & $0.30 \pm 0.04^{* *}$ \\
\hline 8 & Benzoic acid & $0.22 \pm 0.10 * *$ & $0.24 \pm 0.07^{* *}$ \\
\hline 9 & Ferulic acid & $0.27 \pm 0.04^{* *}$ & $0.22 \pm 0.02 * *$ \\
\hline \multirow[t]{2}{*}{10} & Oleacein & $300^{\mathrm{b}} \pm 32$ & $138^{\mathrm{a}} \pm 5$ \\
\hline & $o$-Coumaric acid & n.d. & n.d. \\
\hline 11 & Pinoresinol & $4.02^{b} \pm 0.12$ & $2.64^{\mathrm{a}} \pm 0.07$ \\
\hline 12 & Cinnamic acid & $0.84^{\mathrm{a}} \pm 0.17$ & $0.93^{b} \pm 0.06$ \\
\hline 13 & Apigenin & $0.98^{a} \pm 0.03$ & $1.68^{b} \pm 0.10$ \\
\hline
\end{tabular}

${ }^{*}$ Peak in HPLC chromatogram (Figures 1 and 2). ${ }^{* *}$ Concentration of phenolic compound above limit of detection (LOD) but below limit of quantification (LOQ). n.d.: compound is described as not detected. Different small letters within each row denote significantly different concentrations (ANOVA test, $p \leq 0.05$ ).

Table 2. Concentrations (mean value $\pm \mathrm{SD} ; n=3$ ) of phenolic compounds in VOO (virgin olive oil) and VOO-Sea (virgin seawater-stored olive oil) and $\mathrm{EC}_{50}$ values.

\begin{tabular}{ccc}
\hline & VOO & VOO-Sea \\
\hline Total phenols $^{*}$ & $506^{\mathrm{b}} \pm 63$ & $320^{\mathrm{a}} \pm 2$ \\
$o$-Diphenols & $241^{\mathrm{b}} \pm 58$ & $144^{\mathrm{a}} \pm 9$ \\
Total flavonoids $^{* *}$ & $606^{\mathrm{b}} \pm 71$ & $279^{\mathrm{a}} \pm 53$ \\
EC $_{50}{ }^{* * *}$ & $133^{\mathrm{a}} \pm 11$ & $185^{\mathrm{b}} \pm 15$ \\
\hline
\end{tabular}

* Concentration expressed as mg GAE $/ \mathrm{kg}$ VOO or VOO-Sea \pm SD. ${ }^{* *}$ Concentration expressed as $\mathrm{mg}$ catechin $(\mathrm{CE}) / \mathrm{kg}$ $\mathrm{VOO}$ or VOO-Sea $\pm \mathrm{SD}$. ${ }^{* * *} \mathrm{TP}$ concentration ( $\mu \mathrm{g}$ gallic acid equivalent (GAE)/mL PE or PE-Sea $\pm \mathrm{SD}$ ) required for the reduction of the $50 \%$ of the initial 2,2-diphenyl-1-picrylhydrazyl (DPPH) concentration. Different small letters within each row denote significantly different concentrations (ANOVA test, $p \leq 0.05$ ).

\subsection{Effect of Storage of Olive Fruits in the Seawater on the Phenolic Profile of Olive Oil}

The present study concentrates on the question as to whether the olives contained bioactive phenolic compounds that were eventually preserved or lost during the storage of the fruits in seawater before further processing. Inspection of Tables 1 and 2 and Figure 3 shows that about one half of the phenolics in general were retained in the "seawater olive oil" as compared with the VOO obtained by immediate common mechanical processing of freshly picked olives.

At least with regard to the Oblica variety studied, the most abundant olive variety in Croatia, about two thirds of the total phenolic content as well as the content of the $o$-diphenols were retained in the "seawater oil". In our opinion, there are no obvious reasons to expect a very different pattern with other varieties because we expect the epicarp of olive fruit could be of similar permeability. Similarly, the value of $\mathrm{EC}_{50}$, as one possible measure of antioxidant activity, is increased for VOO-Sea not more than $40 \%$ compared to parent $\mathrm{VOO}$, corresponding to not more than a $40 \%$ decrease in antioxidant activity. The sum of quantified bioactive phenolic compounds (Table 1) is reduced to half (from $334.7 \mathrm{mg} / \mathrm{kg}$ VOO to $165.6 \mathrm{mg} / \mathrm{kg}$ VOO-Sea) but it is important that hydroxytyrosol, biomedically one of the most important phenolic compounds [6] in EVOOs, and tyrosol, known also for beneficial health effects [28], are preserved to 71 and $89 \%$ of the EVOO figure, respectively. Furthermore, there is no phenolic acid or derivative that has been retained less than $58 \%$, except in the case of vanillin where 
$43 \%$ of the content in EVOO remained. However, in the case of homovanillyl alcohol, $p$-coumaric acid and ferulic acid, the corresponding figures are 58,70 and $81 \%$, respectively. Interestingly, the "seawater oil" appears to comprise more vanillic acid than the parent EVOO (50\% increase).

Oleacein, known to have antioxidant, anti-inflammatory, antiproliferative and antimicrobial activities [3,29], is found to be abundant in the examined EVOO of the Oblica variety from the isle of Ugljan; the oil is rich in this hydroxytyrosol derivative, containing $300 \mathrm{mg} / \mathrm{kg}$, see Table 1 . The "seawater oil" contains $138 \mathrm{mg} / \mathrm{kg}, 46 \%$ of the amount in EVOO, nevertheless a significant concentration of oleacein when compared with the literature data for the Oblica variety [22,30]. However, it is necessary to note that the average concentrations of oleacein can reach levels as high as $840 \mathrm{mg} / \mathrm{kg}$, as was detected in some Italian EVOOs [20].

The content of pinoresinol and apigenin [4] has been modified. Moreover, the content of apigenin is $70 \%$ greater in "seawater oil". The results correspond with research conducted by Hachicha et al. [31], whose findings suggest that the apigenin content in olive fruit increases in storage. This suggests that certain biochemical processes in olive fruit are still underway but probably at a slower pace.

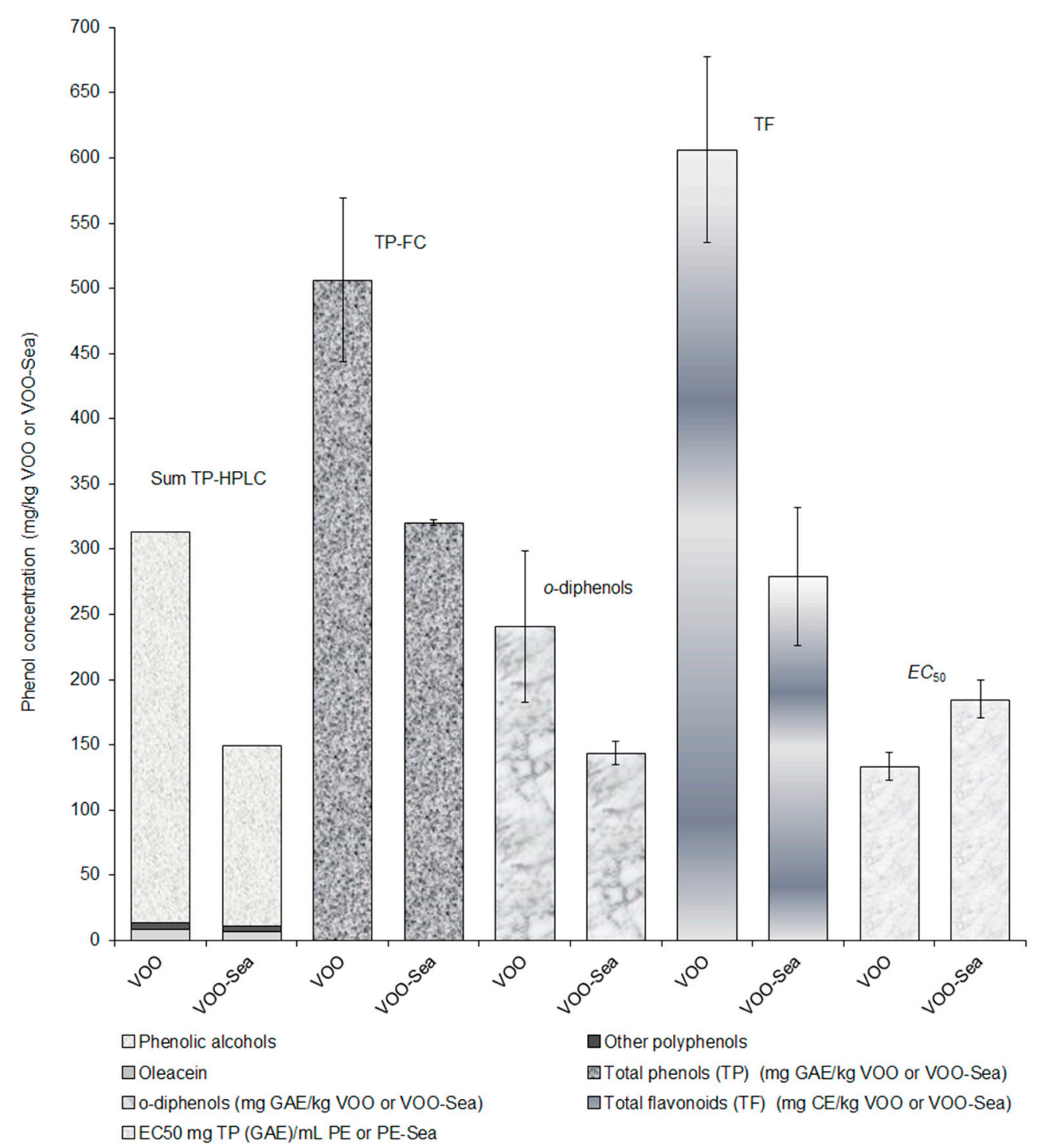

Figure 3. Comparison of phenolic concentrations and $\mathrm{EC}_{50}$ value in VOO and VOO-Sea. Sum TP-HPLC, sum of total phenols determined by HPLC-DAD analysis; TP-FC, total phenols determined by Folin-Ciocalteu analysis; $\mathrm{TF}$, total flavonoids; $\mathrm{EC}_{50}$, concentration of total phenols in PE or PE-Sea leading to $50 \%$ reduction of the initial DPPH concentration. 
Analysis of fragmentation of molecules in ESI-MS spectra confirmed the presence of bioactive secoiridoids: oleacein, oleocanthal, oleuropein aglycone and ligstroside aglycone in VOO and in "seawater olive oil". Oleocanthal especially attracts attention, as it has been compared to ibuprofen [7] and also exerts other biological activities $[3,8]$.

The difference in phenolic profile among VOO and VOO-Sea was moreover confirmed by statistical analysis. Correlation tests reveal different correlation profiles pertaining to a particular olive processing (Tables S3 and S4 in Supplementary Materials). PCA performed on the chemical data shows clustering of the samples on the basis of the effect of storage of olives in seawater prior to processing. Using only three PCs, $97.9 \%$ variance is retained (Figures S3 and S4 in Supplementary Materials).

Although we have determined a loss of phenolic compounds in VOO-Sea, the loss would be even greater in the case of open-air storage [31]. The enzyme activity in the fruit has probably been reduced and some of the oxidation processes have not occurred due to a lower oxygen concentration in the seawater. The presence of pathogenic bacteria and fungi in the fruit is also reduced [32].

Taken together, the comparison between the data for the two olive oils, the VOO obtained from freshly picked and immediately mechanically processed olive fruits of the Oblica variety from Ugljan isle, and the "seawater oil" obtained from the corresponding sample of olive fruits stored in seawater for 10 days prior to common processing, could suggest that despite the reduction in the content of bioactive compounds as a result of storage in seawater, its content is above the values in other varieties of olives and oils. Moreover, when olives of high quality with respect to the content of phenolics are in question, as in the case of the Oblica variety would be expected, the "seawater oils" can be competitive with regard to the biomedical usefulness taking into consideration olive varieties and oils less abundant in the phenolics. In addition, the studies on oil quality have reported no substantial differences with regard to the overall oil quality $[33,34]$. Insofar as the market and the habits of the customers go, "seawater oil" is still very popular.

\section{Conclusions}

The bioactive phenolic compounds in olive oil obtained from fruits stored for ten days in seawater prior to common mechanical processing into oil are partially preserved in the oil. Especially, this should be the case when the olive fruits used are of high quality with respect to the content of the important bioactive phenolics. More favorable results are expected with only several days storage, which is most frequently the case in the actual practice. Therefore, these oils, here denoted as "seawater olive oils", should be considered as oils that are still of some biomedical relevance, although they are of somewhat less biomedical value than the parent EVOOs with high amount of the phenolics possessing favorable healthy effects.

Supplementary Materials: The following are available online at http:/www.mdpi.com/2304-8158/9/10/1347/s1, Figure S1: HPLC-ESI-MS chromatograms (total ion current-TIC) of (a) PE (phenolic extract prepared from VOO); (b) 3,4-DHPEA-EDA (dialdehydic form of decarboxymethyl elenolic acid linked to hydroxytyrosol, oleacein); (c) $p$-HPEA-EDA (dialdehydic form of decarboxymethyl elenolic acid linked to tyrosol, oleocanthal) and p-HPEA-EA (ligstroside aglycone mono-aldehyde); (d) 3,4-DHPEA-EA (oleuropein aglycone mono-aldehyde)., Figure S2: HPLC-ESI-MS chromatograms (total ion current-TIC) of (a) PE-Sea (phenolic extract prepared from VOO-Sea); (b) 3,4-DHPEA-EDA (dialdehydic form of decarboxymethyl elenolic acid linked to hydroxytyrosol, oleacein); (c) $p$-HPEA-EDA (dialdehydic form of decarboxymethyl elenolic acid linked to tyrosol, oleocanthal) and p-HPEA-EA (ligstroside aglycone mono-aldehyde); (d) 3,4-DHPEA-EA (oleuropein aglycone mono-aldehyde),

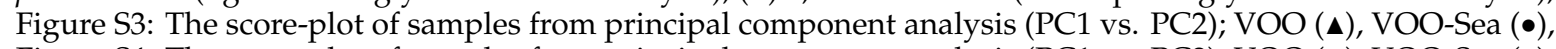
Figure S4: The score-plot of samples from principal component analysis (PC1 vs. PC3); VOO (ی), VOO-Sea $(\bullet)$, Table S1: HPLC-DAD analysis of phenolic compounds in VOO and VOO-Sea, Table S2: Spectrophotometric analysis of phenolic compounds in VOO and VOO-Sea, Table S3: Pearson correlation among individual phenols for VOO samples, ${ }^{*} p<0.05,{ }^{* *} p<0.01$, Table S4: Pearson's correlation among individual phenols for VOO-Sea samples, ${ }^{*} p<0.05,{ }^{* *} p<0.01$.

Author Contributions: Conceptualization, M.B., Đ.B., C.J.B., A.K.M. and S.U.; methodology, M.B., Đ.B., C.J.B. and J.T.; validation, C.J.B., A.K.M. and M.Z.A.; formal analysis, M.B., C.J.B., A.K.M. and J.T.; investigation, M.B., Đ.B., A.K.M., J.T. and S.U.; resources, M.B., C.J.B., Đ.B. and M.Z.A.; data curation, M.B., Đ.B., C.J.B., A.K.M., J.T. and M.Z.A.; writing—original draft preparation, M.B., C.J.B. and S.U.; writing-review \& editing, M.B., Đ.B. 
and C.J.B.; visualization, M.B., A.K.M. and J.T.; supervision, M.B. and S.U.; funding acquisition, M.B., Đ.B., C.J.B., S.U. and M.Z.A. All authors have read and agreed to the published version of the manuscript.

Funding: The Ministry of Science and Education of the Republic of Croatia (006-0063082-0354) provided support for this study.

Acknowledgments: The authors are very grateful to Slaven Dobrović for cooperation. Publication was supported by the OpenAccess Publication Fund of the University of Zagreb Faculty of Agriculture.

Conflicts of Interest: The authors declare no conflict of interest.

\section{References}

1. Ilakovac, B. The Reconstruction of the Olive Mill from the Roman Empire Age at Muline, Isle of Ugljan. Works Inst. Hist. Croat. Acad. Sci. Zadar 1998, 40, 1-26.

2. Torić, J.; Brozović, A.; Baus Lončar, M.; Jakobušić Brala, C.; Karković Marković, A.; Benčić, Đ.; Barbarić, M. Biological activity of phenolic compounds in extra virgin olive oils through their phenolic profile and their combination with anticancer drugs observed in human cervical carcinoma and colon adenocarcinoma cells. Antioxidants 2020, 9, 453.

3. Karković Marković, A.; Torić, J.; Barbarić, M.; Jakobušić Brala, C. Hydroxytyrosol, tyrosol and derivatives and their potential effects on human health. Molecules 2019, 4, 2001.

4. Valls, R.M.; Farras, M.; Suarez, M.; Fernandez-Castillejo, S.; Fito, M.; Konstantinidou, V.; Fuentes, F.; López-Miranda, J.; Giralt, M.; Covas, M.I.; et al. Effects of functional olive oil enriched with its own phenolic compounds on endothelial function in hypertensive patients. A randomised controlled trial. Food Chem. 2015, 167, 30-35.

5. Celano, M.; Maggisano, V.; Lepore, S.M.; Russo, D.; Bulotta, S. Secoiridoids of olive and derivatives as potential coadjuvant drugs in cancer: A critical analysis of experimental studies. Pharmacol. Res. 2019, 142, 77-86.

6. Wani, T.A.; Masoodi, F.A.; Gani, A.; Baba, W.N.; Rahmanian, N.; Wani, I.A.; Ahmad, M. Olive oil and its principal bioactive compound: Hydroxytyrosol-A review of the recent literature. Trends Food Sci. Technol. 2018, 77, 77-90.

7. Beauchamp, G.K.; Keast, R.S.J.; Morel, D.; Lin, J.; Pika, J.; Han, Q.; Lee, C.L.; Smith, A.B.; Breslin, P.A.S. Ibuprofen-like activity in extra-virgin olive oil: Phytochemistry. Nature 2005, 437, 45-46.

8. Parkinson, L.; Keast, R. Oleocanthal, a Phenolic Derived from Virgin Olive Oil: A Review of the Beneficial Effects on Inflammatory Disease. Int. J. Mol. Sci. 2014, 15, 12323-12334.

9. Miljković, I. Modern Fruit Growing; Znanje: Zagreb, Croatia, 1991.

10. Jerman Klen, T.; Mozetič Vodopivec, B. Optimisation of olive oil phenol extraction conditions using a high-power probe ultrasonication. Food Chem. 2012, 134, 2481-2488.

11. Jakobušić Brala, C.; Benčić, D.; Šindrak, Z.; Barbarić, M.; Uršić, S. Labeled extra virgin olive oil as food supplement; phenolic compounds in oils from some autochthonous Croatian olives. Grasas Aceites 2015, 66, e099.

12. Pampaloni, B.; Mavilia, C.; Fabbri, S.; Romani, A.; Ieri, F.; Tanini, A.; Tonelli, F.; Brandi, M.L. In Vitro Effects of Extracts of Extra Virgin Olive Oil on Human Colon Cancer Cells. Nutr. Cancer 2014, 66, 1228-1236. [CrossRef] [PubMed]

13. Gutfinger, T. Polyphenols in olive oils. J. Am. Oil Chem. Soc. 1981, 58, 966-968.

14. Mateos, R.; Espartero, J.L.; Trujillo, M.; Ríos, J.J.; León-Camacho, M.; Alcudia, F.; Cert, A. Determination of phenols, flavones, and lignans in virgin olive oils by solid-phase extraction and high-performance liquid chromatography with diode array ultraviolet detection. J. Agric. Food Chem. 2001, 49, 2185-2192.

15. Kim, D. Antioxidant capacity of phenolic phytochemicals from various cultivars of plums. Food Chem. 2003, 81, 321-326.

16. Villaño, D.; Fernández-Pachón, M.S.; Moyá, M.L.; Troncoso, A.M.; García-Parrilla, M.C. Radical scavenging ability of polyphenolic compounds towards DPPH free radical. Talanta 2007, 71, 230-235.

17. Antonini, E.; Farina, A.; Leone, A.; Mazzara, E.; Urbani, S.; Selvaggini, R.; Servili, M.; Ninfali, P. Phenolic compounds and quality parameters of family farming versus protected designation of origin (PDO) extra-virgin olive oils. J. Food Compost. Anal. 2015, 43, 75-81.

18. Jerman Klen, T.; Golc Wondra, A.; Vrhovšek, U.; Mozetič Vodopivec, B. Phenolic profiling of olives and olive oil process-derived matrices using UPLC-DAD-ESI-QTOF-HRMS analysis. J. Agric. Food Chem. 2015, 63, 3859-3872. 
19. Becerra-Herrera, M.; Velez-Martin, A.; Ramos-Merchante, A.; Richter, P.; Beltran, R.; Sayago, A. Characterization and evaluation of phenolic profiles and color as potential discriminating features among Spanish extra virgin olive oils with protected designation of origin. Food Chem. 2018, 241, 328-337.

20. Fanali, C.; Della Posta, S.; Vilmercati, A.; Dugo, L.; Russo, M.; Petitti, T.; Mondello, L.; de Gara, L. Extraction, analysis, and antioxidant activity evaluation of phenolic compounds in different italian extra-virgin olive oils. Molecules 2018, 23, 3249.

21. Kulišić Bilušić, T.; Melliou, E.; Giacometti, J.; Čaušević, A.; Čorbo, S.; Landeka, M.; Magiatis, P. Phenolics, fatty acids, and biological potential of selected Croatian EVOOs: Characterization of selected Croatian EVOOs. Eur. J. Lipid Sci. Technol. 2017, 119, 1700108.

22. Šarolić, M.; Gugić, M.; Friganović, E.; Tuberoso, C.; Jerković, I. Phytochemicals and other characteristics of croatian monovarietal extra virgin olive oils from oblica, lastovka and levantinka varieties. Molecules 2015, 20, 4395-4409. [CrossRef] [PubMed]

23. Franco, M.N.; Galeano-Diaz, T.; Sanchez, J.; de Miguel, C.; Martin-Vertedor, D. Antioxidant capacity of the phenolic fraction and its effect on the oxidative stability of olive oil varieties grown in the southwest of Spain. Grasas Aceites 2014, 65, e004.

24. Rotondi, A.; Fabbri, A.; Tommaso, G. Sensory and chemical properties of extra virgin olive oils produced in two different Italian regions: Tuscany and Emilia-Romagna. J. Food Agric. Environ. 2008, 6, 71-77.

25. Kosma, I.; Vatavali, K.; Kontakos, S.; Kontominas, M.; Kiritsakis, A.; Badeka, A. Geographical differentiation of Greek extra virgin olive oil from late-harvested Koroneiki cultivar fruits. J. Am. Oil Chem. Soc. 2017, 94, 1373-1384. [CrossRef]

26. Ocakoglu, D.; Tokatli, F.; Ozen, B.; Korel, F. Distribution of simple phenols, phenolic acids and flavonoids in Turkish monovarietal extra virgin olive oils for two harvest years. Food Chem. 2009, 113, 401-410. [CrossRef]

27. Rigane, G.; Ayadi, M.; Boukhris, M.; Sayadi, S.; Bouaziz, M. Characterisation and phenolic profiles of two rare olive oils from southern Tunisia: Dhokar and Gemri-Dhokar cultivars. J. Sci. Food Agric. 2013, 93, 527-534. [CrossRef]

28. Rodríguez-Morató, J.; Boronat, A.; Kotronoulas, A.; Pujadas, M.; Pastor, A.; Olesti, E.; Pérez-Mañá, C.; Khymenets, O.; Fitó, M.; Farré, M.; et al. Metabolic disposition and biological significance of simple phenols of dietary origin: Hydroxytyrosol and tyrosol. Drug Metab. Rev. 2016, 48, 218-236. [CrossRef]

29. Cuyas, E.; Verdura, S.; Lozano-Sanchez, J.; Viciano, I.; Llorach-Pares, L.; Nonell-Canals, A.; Bosch-Barrerab, J.; Brunet, J.; Segura-Carreteroc, A.; Sanchez-Martineze, M.; et al. The extra virgin olive oil phenolic oleacein is a dual substrate-inhibitor of catechol-O-methyltransferase. Food Chem. Toxicol. 2019, 128, 35-45. [CrossRef]

30. Bilušić, T.; Žanetić, M.; Ljubenkov, I.; Generalić Mekinić, I.; Štambuk, S.; Bojović, V.; Soldo, B.; Magiatis, P. Molecular characterization of Dalmatian cultivars and the influence of the olive fruit harvest period on chemical profile, sensory characteristics and oil oxidative stability. Eur. Food Res. Technol. 2018, 244, 281-289. [CrossRef]

31. Hachicha Hbaieb, R.; Kotti, F.; García-Rodríguez, R.; Gargouri, M.; Sanz, C.; Pérez, A.G. Monitoring endogenous enzymes during olive fruit ripening and storage: Correlation with virgin olive oil phenolic profiles. Food Chem. 2015, 174, 240-247. [CrossRef]

32. Clodoveo, M.L.; Delcuratolo, D.; Gomes, T.; Colelli, G. Effect of different temperatures and storage atmospheres on Coratina olive oil quality. Food Chem. 2007, 102, 571-576. [CrossRef]

33. Koprivnjak, O.; Procida, G.; Bencic, D.; Zelinotti, T. Effect of olive fruits storage in sea water on oil quality. Food Technol. Biotechnol. 1999, 37, 209-214.

34. Koprivnjak, O.; Procida, G.; Zelinotti, T. Changes in the volatile components of virgin olive oil during fruit storage in aqueous media. Food Chem. 2000, 70, 377-384. [CrossRef]

(C) 2020 by the authors. Licensee MDPI, Basel, Switzerland. This article is an open access article distributed under the terms and conditions of the Creative Commons Attribution (CC BY) license (http://creativecommons.org/licenses/by/4.0/). 